\title{
EXPANSIONS OF THE IRREGULAR COULOMB FUNCTION*
}

\author{
BY \\ FRANK S. HAM** \\ Department of Physics, Harvard University
}

\begin{abstract}
Convergent and asymptotic series in the energy are derived for an irregular Coulomb function appropriate to both attractive and repulsive potentials. The coefficients in these series are Bessel functions. For repulsive potentials the derivation yields series in agreement with those given by Breit and others. Some of the series for attractive potentials have been given by Kuhn. The present general derivation and some of the series are, however, new. Tables of the coefficients in the series for the attractive potentials for values of the parameters of interest in solid state energy band calculations have been prepared and are available.
\end{abstract}

I. Introduction. Breit and $\mathrm{Hull}^{1}$ and Abramowitz ${ }^{2}$ have shown that the irregular Coulomb function $G_{L}$ can be expanded in an asymptotic power series in the energy which is valid for repulsive Coulomb fields. Jackson and Blatt ${ }^{3}$ have given a convergent series of similar form for $G_{0}$, and their derivation can evidently be extended to higher $L$. In the present note we shall derive convergent and asymptotic series for an irregular function appropriate to both attractive and repulsive potentials and shall obtain the results of the above authors as a special case. A review of the numerical treatment of Coulomb functions has been given recently by Fröberg (Rev. Mod. Phys. 27, 399 (1955)).

The investigation leading to these general results was undertaken in order to establish formulas for the convenient numerical calculation of an irregular function for attractive Coulomb fields and positive and negative energies of small absolute value. Such calculations are an essential preliminary to the theoretical investigation with the "Quantum Defect Method" of the energy band structure of the alkali metals, a program of research which is being continued by Brooks and the author. Therefore, we shall use a notation in this paper which is most appropriate to work with an attractive Coulomb field, but in an appendix we shall show the connections with the notation used by Breit and others in their work with a repulsive field and shall show that our results agree with theirs.

$\mathrm{Kuhn}^{5}$ has already given the asymptotic series that will be derived in this paper, but his presentation is marred by the fact that he did not realize that these irregular series do not converge. Our convergent series are, however, new. We shall assume that the reader is familiar with Kuhn's paper.

*Received January 18, 1956. This paper is based upon a section of a thesis submitted by the author to the Faculty of Harvard University in partial fulfillment of the requirements for the degree of Doctor of Philosophy.

**Present address: General Electric Research Laboratory, Schenectady, N. Y.

'G. Breit and M. H. Hull, Phys. Rev. 80, 392, 561 (1950); G. Breit and W. G. Bouricius, Phys. Rev. 75, 1029 (1949)

2M. Abramowitz, J. Math. and Phys. 33, 111 (1954)

J. D. Jackson and J. M. Blatt, Rev. Mod. Phys. 22, 77 (1950)

H. Brooks and F. S. Ham, Phys. Rev. (to be published)

T. S. Kuhn, Quart. Appl. Math. 9, No. 1 (1951) 
A set of tables of the coefficients in the series for the regular and irregular functions for the attractive Coulomb field has been prepared for values of the parameters of interest in the solid state calculations. These extend and correct the tables given by $\mathrm{Kuhn}^{5}$ and are accompanied by a more detailed discussion of the formulas than can be given here. They contain also tab!es of various quantities which appear in the formulas and which will not be written out explicitly here. These tables are now available and may be obtained from the author or from the Division of Applied Science, Harvard University".

II. Convergent series representations of Coulomb functions for non-integral values of $(2 L+1)$. The hydrogenic radial wave equation in Rydberg units for an attractive potential takes the form ${ }^{s}$

$$
\frac{d^{2} U}{d r^{2}}+\left[-\frac{1}{n^{2}}+\frac{2}{r}-\frac{L(L+1)}{r^{2}}\right] U=0,
$$

where $r$ is the radial coordinate, $U$ the radial part of the wave function multiplied by $r, L$ the angular momentum quantum number, and $E=-1 / n^{2}$ the energy. Yost, Wheeler and Breit and $\mathrm{Kuhn}^{\mathrm{b}}$ have shown that solutions of (1) exist which have the form

$$
U^{(L, n)}(z)=\sum_{k=0}^{\infty} n^{-2 k} U_{k}^{(L)}(z),
$$

where $z=(8 r)^{1 / 2}$. If we set

$$
U_{k}^{(L)}(z)=(z / 2) V_{k}^{(L)}(z),
$$

we find that the $V_{k}^{(L)}(z)$ satisfy the simultaneous differential equations

where

$$
\begin{gathered}
\nabla_{L} V_{0}^{(L)}(z)=0, \\
\nabla_{L} V_{k}^{(L)}(z)=(z / 2)^{4} V_{k-1}^{(L)}(z),
\end{gathered}
$$

$$
\nabla_{L}=z^{2}\left(\frac{d^{2}}{d z^{2}}\right)+z\left(\frac{d}{d z}\right)+z^{2}-(2 L+1)^{2} .
$$

Kuhn has shown from the recurrence relations satisfied by the Bessel functions that if $C_{m}(z)$ is a linear combination of the Bessel function $J_{m}(z)$ and the Weber function $Y_{m}(z)$

$$
C_{m}(z)=A J_{m}(z)+B Y_{m}(z),
$$

$A$ and $B$ being independent of $m$, then

$$
\begin{aligned}
\nabla_{L}\left\{\frac{2 L+2+q}{4(2+q)}(z / 2)^{e+2} C_{2 L+8+q}(z)-\frac{1}{4(3+q)}(z / 2)^{a+3} C_{2 L+4+e}(z)\right\} & \\
& =(z / 2)^{e+4} C_{2 L+1+e}(z),
\end{aligned}
$$

and solutions of (4) may be generated if we choose

$$
V_{0}^{(L)}(z)=C_{2 L+1}(z) \text {. }
$$

T. S. Ham, Tables for the calculation of Coulomb rave functions, Technical Report No. 204, Cruft Laboratory, Harvard University

T. L. Yost, J. A. Wheeler and G. Breit, Phys. Rev. 49, 174 (1936) 
In particular, if in (6) we choose $A=1, B=0$, and if we then determine $V_{k}^{(L)}(z)$ from (7) for $k \geq 1$, so that no $V_{k}^{(L)}(z)$ for $k \geq 1$ when expanded in powers of $(z / 2)$ contains the power $(z / 2)^{2 L+1}$, we obtain the series

$$
J_{2 L+1}^{n}(z)=(2 / z)^{0} U^{(L, n)}(z)=\sum_{k=0}^{\infty} n^{-2 k} \sum_{a-3 k}^{3 k} a_{k, Q}^{(L)}(z / 2)^{\natural} J_{2 L+1+e}(z) .
$$

It is shown in Appendix I that this series converges absolutely and uniformly for $|z| \leq R$ and $|n| \geq n_{0}$, where $R$ and $n_{0}$ are arbitrary positive numbers.

Similarly, we may show that

$$
\begin{aligned}
\nabla_{L}\left\{\frac{-2 L+q}{4(2+q)}\left(\frac{z}{2}\right)^{a+2} C_{-(2 L+1)+a+2}(z)-\frac{1}{4(q+3)}\left(\frac{z}{2}\right)^{a+3} C_{-(2 L+1)+a+3}(z)\right\} & \\
& =\left(\frac{z}{2}\right)^{a+4} C_{-(2 L+1)+Q}(z) .
\end{aligned}
$$

Choosing $V_{0}^{(L)}(z)=J_{-(2 L+1)}(z)$, we obtain the series

$$
J_{-(2 L+1)}^{n}(z)=\sum_{k=0}^{\infty} n^{-2 k} \sum_{a=2 k}^{3 k} b_{k, e}^{(L)}(z / 2)^{\bullet} J_{-(2 L+1)+a}(z),
$$

in which the $b_{k, a}^{(L)}$ are determined from (10) in the same manner as the $a_{k, e}^{(L)}$ are obtained from (7), so that $(z / 2)^{-(2 L+1)}$ occurs only in the term $V_{0}^{(L)}(z)$. In the following discussion we shall always mean by $a_{k, e}^{(L)}$ and $b_{k, e}^{(L)}$ the coefficients appearing in these series, determined as described above. The proof that (11) converges absolutely and uniformly for $|z| \leq R$ and $|n| \geq n_{0}$ is carried out in a manner identical with the proof of the convergence of (9).

Since both $(z / 2) J_{2 L+1}^{n}(z)$ and the regular Whittaker function ${ }^{8} M_{n, L+1}\left(z^{2} / 4 n\right)$ satisfy (1), it follows by comparison of the coefficients of $(z / 2)^{2 L+2}$ in the series expansions of both functions that ${ }^{5}$

$$
J_{2 L+1}^{n}(z)=\left[\frac{n^{L+1}}{(z / 2) \Gamma(2 L+2)}\right] M_{n, L+1 / 2}\left(z^{2} / 4 n\right) .
$$

Similarly, if $(2 L+1)$ is not an integer, we may show that $(z / 2) J_{-(2 L+1)}^{n}(z)$ is related to $M_{n,-L-1}\left(z^{2} / 4 n\right)$ by a formula obtained from (12) by replacing $L$ by $-L-1$. Hence if $(2 L+1)$ is not an integer, (9) and (11) are two independent solutions of (1), both of which are satisfactorily convergent in both $z$ and $1 / n$. Kuhn has proved the existence of two such series solutions ${ }^{5}$ and has exhibited (9); however, instead of (11) he supposed that the series obtained from (9) by replacing $J_{m}(z)$ by $Y_{m}(z)$ converged, whereas in fact the resulting series diverges, as we shall show.

If $(2 M+1)$ is a positive integer, the function $M_{n,-M-1}\left(z^{2} / 4 n\right)$ is not defined, and we may show that ${ }^{\circ}$

$$
\lim _{L \rightarrow M} J_{-2 L-1}^{n}(z)=\frac{\Gamma(n+M+1) \cos (2 M+1) \pi}{n^{2 . M+1} \Gamma(n-M)} J_{2 M+1}^{n}(z) .
$$

${ }^{8} \mathrm{E}$. T. Whittaker and G. X. Watson, $A$ course of modern analysis, 4th ed., Cambridge Univ. Press, 1952, p. 337

${ }^{9}$ G. H. Wannier, Phy's. Rev. 64, 358 (1943) 
Hence in order to obtain a second solution of (1) which is independent of $J_{2 L+1}^{n}(z)$ for all values of $2 L+1$, we define ${ }^{0}$

$N_{2 L+1}^{n}(z)=\left[\frac{\Gamma(n+L+1)}{n^{2 L+1} \Gamma(n-L)} J_{2 L+1}^{n}(z) \cos (2 L+1) \pi-J_{-2 L-1}^{n}(z)\right]\left(\frac{1}{\sin (2 L+1) \pi}\right)$

and understand that, for integral values of $(2 L+1), N_{2 L+1}^{n}(z)$ is defined by the limit of the expression on the right of (14) as $(2 L+1) \rightarrow$ integer.

III. Series representations of $N_{2 L+1}^{n}(z)$. To derive a convergent representation of $N_{2 L+1}^{n}(z)$ in a power series in $\left(1 / n^{2}\right)$ we may substitute (9) and (11) into (14), since both of these series converge absolutely for all $L$. However, for non-integral values of $(2 L+1)$ we cannot expand the coefficient of $J_{2 L+1}^{n}(z)$ in (14) in a convergent power series in $\left(1 / n^{2}\right)$ because $\Gamma(n+L+1)$ has a pole whenever $(n+L+1)$ is a negative integer or zero. We therefore write (14) as

$$
\begin{aligned}
& N_{2 L+1}^{n}(z) \sin (2 L+1) \pi=\left[\frac{\Gamma(n+L+1)}{n^{2 L+1} \Gamma(n-L)}-1\right] \text {. } \\
& \cdot \sum_{k=0}^{\infty} n^{-2 k} \sum_{a-2 k}^{3 k} a_{k, e}^{(L)}(z / 2)^{e} J_{2 L+1+e}(z) \cos (2 L+1) \pi \\
& +\sum_{k=0}^{\infty} n^{-2 k}\left\{\sum_{a=2 k}^{3 k} a_{k, 0}^{(L)}(-)^{e}(z / 2)^{e} J_{2 L+1+e}(z) \cos (2 L+1+q) \pi\right. \\
& \left.-\sum_{a=2 k}^{3 k} b_{k \cdot 0}^{(L)}(z / 2)^{\bullet} J_{-(2 L+1)+e}(z)\right\} \text {. }
\end{aligned}
$$

The $k=0$ term of the second series in (15) is seen to be $\sin (2 L+1) \pi Y_{2 L+1}(z)$, since $a_{00}^{(L)}=b_{00}^{(L)}=1$. Since the first series in (15) is simply a multiple of $J_{2 L+1}^{n}(z)$, and because both $(z / 2) J_{2 L+1}^{n}(z)$ and $(z / 2) N_{2 L+1}^{n}(z)$ satisfy (1), we see from the form of (15), Eq. (7), and the above value of the $k=0$ term that the $k=1$ term of the second series in (15) is determined by (4) to be

$$
n^{-2}\left[a_{1,2}^{(L)}(z / 2)^{2} Y_{2 L+8}(z)+a_{1,3}^{(L)}(z / 2)^{8} Y_{2 L+4}(z)\right] \sin (2 L+1) \pi
$$

plus some linear combination of the solutions of the homogeneous equation (4a), $J_{2 L+1}(z)$ and $J_{-2 L-1}(z)$. We can determine this latter combination by using the Bessel function recurrence relations to re-express the terms involving $\left(1 / n^{2}\right) J_{-2 L-1+e}(z)$ in the second series. We see that we shall obtain

$$
\begin{aligned}
-b_{1,2}^{(L)}(z / 2)^{2} J_{-2 L-1+2}(z)-b_{1,3}^{(L)}(z / 2)^{3} J_{-2 L-1+3}(z) & \\
& =-a_{1,2}^{(L)}(z / 2)^{2} J_{-2 L-3}(z)+a_{1,3}^{(L)}(z / 2)^{3} J_{-2 L-4}(z) \\
& -A(L) J_{-2 L-1}(z)-B(L) J_{2 L+1}(z) .
\end{aligned}
$$

We need not carry this out explicitly in order to obtain the polynomials $A(L)$ and $B(L)$, however. Because the recurrence relations relate Bessel functions of orders differing by integers, if $2(2 L+1)$ is not an integer ${ }^{10}$ we shall not obtain $J_{2 L+1}(z)$ in an expression for $J_{-2 L-1+a}(z)$, so that $B(L)=0$. We may obtain $A(L)$ if we notice that when $2 L+1$ equals an integer, $2 M+1$, the left side of (15) vanishes for all $n$ and that

\footnotetext{
${ }^{10}$ We make use of non-integral values of $2(2 L+1)$ in order to prove $B(L)=0$. Both sides of (17) being continuous functions of $L$, the conclusion holds for $2(2 L+1)=$ integer as well.
} 


$$
\frac{\Gamma(M+n+1)}{n^{2 M+1} \Gamma(n-M)}=\sum_{p=0}^{[M+1]} \frac{b_{p}(M)}{n^{2 p}},
$$

the $b_{3}(M)$ being polynomials the calculation of which is outlined in Appendix II, and $[M+1]$ denoting the largest integer less than $M+1$ (i.e. $[M+1]$ equals $M$ if $M$ is an integer, $\left(M+\frac{1}{2}\right)$ if $M$ is half an odd integer). Therefore the coefficients of $\left(1 / n^{2}\right)$ on the right of (15) must vanish when $L=M$, and since $J_{-2 M-1}(z)=(-1)^{2 N+1} J_{2 M+1}(z)$, we find on combining all terms in $1 / n^{2}$ in (15) when $L=M$ and using (16), (17), and (18), that $A(L)$ must differ from $b_{1}(L)$ by at most a function of $L$ that vanishes for all $M$. Since $A(L)$ is itself a polynomial, however, we have that $A(L)=b_{1}(L)$.

Continuing this argument to higher powers of $\left(1 / n^{2}\right)$, we find that the desired form of the series is given us immediately: the term in $n^{-2 k}$ is determined from that for $n^{-2(k-1)}$ by the use of the differential equations (4) and (7) except for a multiple of $n^{-2 k} J_{-2 L-1}(z)$; this multiple is determined from the requirement that this term cancels for all integral values of $2 M+1$ the term $-n^{-2 k} b_{k}(M) J_{2 M+1}(z)$ in the first series of (15).

We now wish to use this information to rewrite (15) in a form such that when $L=M$ each term of the series vanishes. We can't do this, once and for all, for arbitrary $M$ because, as we shall see, we should have to make use of a divergent asymptotic series which we want to avoid. But if we are interested in a particular $M$ we can conveniently proceed as follows. We know that the series (9) and (11) converge absolutely, so that we can add to the second series in (15) a finite polynomial in powers of $\left(1 / n^{2}\right)$ multiplied by (9) and rearrange the resulting combined series in powers of $\left(1 / n^{2}\right)$ without disturbing convergence. Choosing this polynomial to be $\sum_{p=1}^{[M+1]} b_{p}(L) n^{-2 p}$, adding it in as described and subtracting it out in combination with the first series, we have finally on making use of the relation $b_{0}(L)=1$

$$
\begin{aligned}
& N_{2 L+1}^{n}(z)=\left[\frac{\Gamma(L+n+1)}{n^{2 L+1} \Gamma(n-L)}-\sum_{p=0}^{[N+1]} \frac{b_{p}(L)}{n^{2 p}}\right] \frac{\cos (2 L+1) \pi}{\sin (2 L+1) \pi} J_{2 L+1}^{n}(z) \\
& +\sum_{k=0}^{(k+1)} n^{-2 k} \sum_{p=0}^{k} b_{p}(L) \sum_{e-2(k-p)}^{3(k-p)} a_{k-p, e}^{(L)}(z / 2)^{e} Y_{2 L+1+e}(z) \\
& +\sum_{k=\{L+1]+1}^{\infty} n^{-2 k}\left\{\sum_{p=0}^{[M+1]} b_{p}(L) \sum_{e-2(k-p)}^{3(k-p)} a_{k-p, e}^{(L)}(z / 2)^{e} Y_{2 L+1+a}(z)\right. \\
& \left.-\sum_{p-\{k+1\}+1}^{k}\left[\frac{b_{p}(L)}{\sin (2 L+1) \pi}\right]_{a=2(k-p)}^{3(k-p)} a_{k-p, e}^{(L)}(-)^{q}(z / 2)^{\natural} J_{-(2 L+1+q)}(z)\right\} \text {. }
\end{aligned}
$$

We see that the series is now in a particularly convenient form for taking the limit $L \rightarrow M$, where, we recall, $2 M+1$ is a particular integer. Thus using (18) we can write for $L \rightarrow M$

$$
\begin{aligned}
G_{M}(M, n) & =\lim _{L \rightarrow M} \frac{\cos (2 L+1) \pi}{\sin (2 L+1) \pi}\left\{\frac{\Gamma(L+n+1)}{n^{2 L+1} \Gamma(n-L)}-\sum_{p=0}^{[M+1]} \frac{b_{p}(L)}{n^{2 p}}\right\} \\
& =\frac{1}{2 \pi}\left\{\frac{d}{d L}\left(\frac{\Gamma(n+L+1)}{n^{2 L+1} \Gamma(n-L)}\right)-\sum_{p=0}^{[M+1]} n^{-2 p} \frac{d}{d L}\left(b_{p}(L)\right)\right\}_{L-M} \\
& =\frac{1}{2 \pi}\left\{\frac{\Gamma(L+n+1)}{n^{2 L+1} \Gamma(n-L)}[\Psi(n+L+1)+\Psi(n-L)-2 \ln (n)]\right. \\
& \left.-\sum_{p=0}^{\{M+1\}} \frac{1}{n^{2 p}} \frac{d}{d L}\left(b_{p}(L)\right)\right\}_{L-M}
\end{aligned}
$$


where $\Psi(x)=(d / d x) \ln \Gamma(x)$. Also it is in general true that $b_{p}(M)=0$ if $p>[M+1]$. Thus all terms in (19) vary continuously as $L \rightarrow M$.

We notice in (19) that $(z / 2) N_{2 L+1}^{n}(z)$ has been expressed as a multiple $G_{M}(L, n)$ of the regular function $(z / 2) J_{2 L+1}^{n}(z)$ plus an absolutely convergent series in $\left(1 / n^{2}\right)$ which we shall call $(z / 2) Q_{2 L+1}^{n}(z, M)$ and which is clearly an irregular solution of (1). Both $(z / 2) N_{2 L+1}^{n}(z)$ and $(z / 2) Q_{2 L+1}^{*}(z, M)$ satisfy a Wronskian relation with $(z / 2) J_{2 L+1}^{n}(z)$ :

$\left[(z / 2) J_{2 L+1}^{n}(z)\right]\left[(z / 2)(d / d z)(z / 2) N_{2 L+1}^{n}(z)\right]$

$$
\text { - }\left[(z / 2) N_{2 L+1}^{n}(z)\right]\left[(z / 2)(d / d z)(z / 2) J_{2 L+1}^{n}(z)\right]=z^{2} / 4 \pi .
$$

The relation involving $Q_{2 L+1}^{n}(z, M)$ is identical with (21) if $N$ is replaced by $Q$. A number of other formulas involving $N_{2 L+1}^{n}(z)$ are given in Appendix III.

An asymptotic series for $N_{2 L+1}^{*}(z)$ in powers of $\left(1 / n^{2}\right)$ for arbitrary $L$ may be obtained from the convergent representation (19) if we make use of the asymptotic expansion (A3) (Appendix II), valid for $|\arg (n)| \leq \pi-\Delta<\pi$, and rearrange (19), making use of the fact that we may multiply and add asymptotic series. Combining terms in $J_{2 L+1+q}(z)$ and $J_{-2 L-1-q}(z)$ by means of the definition ${ }^{11}$ of the Weber function, we obtain

$$
N_{2 L+1}^{n}(z) \underset{1 n ! \rightarrow \infty}{\sim} \sum_{p=0}^{\infty} n^{-2 p} b_{p}(L) \sum_{k=0}^{\infty} n^{-2 k} \sum_{a=2 k}^{8 k} a_{k, \Theta}^{(L)}(z / 2)^{a} Y_{2 L+1+a}(z) .
$$

Multiplying by the reciprocal expansion of (A3), we have also

$$
\frac{\Gamma(n-L) n^{2 L+1}}{\Gamma(n+L+1)} N_{2 L+1}^{n}(z) \underset{|x| \rightarrow \infty}{\sim} \sum_{k=0}^{\infty} n^{-2 k} \sum_{a=2 k}^{3 k} a_{k, a}^{(L)}(z / 2)^{a} Y_{2 L+1+e}(z) .
$$

Except when $(2 L+1)$ equals half an odd integer, both (22) and (23) may be proved to be divergent series ${ }^{6}$ which are asymptotic representations in $n$, for $|\arg (n)| \leq$ $\pi-\Delta<\pi$, of the functions on the left of the equations. We note that (23) has the same form as the convergent series (9) but with $Y_{m}(z)$ replacing $J_{m}(z){ }^{12}$

IV. Acknowledgments. The author would like to thank Professor Harvey Brooks for discussing this work with him and for making several helpful criticisms.

Appendix I. Proof of the convergence of (9). It follows from (7) that the numbers $a_{k, e}^{(L)}$ have an upper bound $\beta^{(L)}$. From the series definition of the Bessel function $J_{m}(z)$ we may show that

$$
\left|J_{m}(z)\right|<(|z| / 2)^{m}(m !)^{-1} \exp \left(|z|^{2} / 4\right)
$$

Since $2 k \leq q \leq 3 k$ in (9), by writing (9) in the form (2) we may show that, if $k_{0}$ is sufficiently large, then for all $k \geq k_{0}$,

$$
\left|{ }^{0} U_{k \bullet}^{(L)}(z) n^{-2 k}\right|<\beta^{(L)} \exp \left(|z|^{2} / 4\right)(|z| / 2)^{2 L+4 k+2}|n|^{-2 k}[(2 L+2 k) !]^{-1} .
$$

If we replace $|z|$ by $R$ and $|n|$ by $n_{0}$ on the right of (A2), where $R$ and $n_{0}$ are arbitrary positive numbers, then the series in $k$ formed from the resulting quantities can be shown

"Whittaker and Watson, op. cit. p. 370

"The series (23) is one of those given by Kuhn in Footnote 5 . We have also verified for $L=0$, 1, 2, 3 that Kuhn's alternate generating procedure with $C_{0}(z)=Y_{0}(z)$ and $C_{1}(z)=Y_{1}(z)$ yields the asymptotic series (22) for $(z / 2) N_{2 L+1}^{n}(z)$ for these values of $L$, for which $b_{p}(L)=0$ when $p \geq L+1$. Hence Kuhn's tables for the irregular function are convenient if the asymptotic representation is sufficiently accurate. 
to converge absolutely by the ratio test. It therefore follows from (A2), the comparison test, and the Weierstrass $M$-test that the original series (9) converges absolutely and uniformly for $|z| \leq R,|n| \geq n_{0}$.

Appendix II. The polynomials $b_{p}(M)$. From the exponential form of Stirling's series ${ }^{13}$ for $\Gamma(x)$, we may show that for an arbitrary $L$ the following asymptotic relation holds for $|\arg (n)| \leq \pi-\Delta<\pi$ as $|n| \rightarrow \infty$ :

$$
\frac{\Gamma(n+L+1)}{n^{2 L+1} \Gamma(n-L)} \sim \sum_{p=0}^{\infty} \frac{b_{p}(L)}{n^{2 p}}
$$

the $b_{p}(L)$ being polynomials in $L$. In order to determine these, we may let $L$ equal an integer $M$. Then from the relation $\Gamma(x+1)=x \Gamma(x)$ we may evaluate the left side of (A3), obtaining

$$
n^{-2 . M}\left(n^{2}-M^{2}\right)\left(n^{2}-(M-1)^{2}\right) \cdots\left(n^{2}-1\right)=\sum_{p=0}^{M} b_{p}(M) n^{-2 p} .
$$

Clearly $b_{p}(L)$ vanishes if $L$ equals an integer less than $p$. It also vanishes if $L$ is a half integer less than $p-1$. We see from (A4) that $b_{p}(M)$ for integral $M$ is equal to $(-1)^{p}$ times the sum of all different products of the squares of $p$ integers less than or equal to $M$, no two integers in any product being the same. This may be written as

$$
b_{p}(M)=(-)^{p} \sum_{k_{p}-p}^{M} k_{p}^{2} \sum_{k_{p-1}-p-1}^{k_{p-1}-1} k_{p-1}^{2} \sum_{k_{p-s}-p-2}^{k_{p-1}-1} k_{p-2}^{2} \cdots \sum_{k_{1}-1}^{k_{2}-1} k_{1}^{2} \text {. }
$$

Therefore

$$
b_{p}(M)=-\sum_{k_{p}-p}^{M} k_{p}^{2} b_{p-1}\left(k_{p}-1\right) \text {. }
$$

The relation (A6), the fact that $b_{0}(M)=1$, and the formula

$$
\sum_{k=1}^{N} k(k+1)(k+2) \cdots(k+n)=M(M+1)(M+2) \cdots(M+n+1)(n+2)^{-1}
$$

may be used to calculate the $b_{p}(M)$ as polynomials in $M$. Since the resulting polynomials are correct for all integral values of $M \geq p$, we can replace $M$ by $L$ in order to obtain the $b_{p}(L)$ appropriate to (A3) for general values of $L$. These polynomials are given in the reference of Footnote 6 for $p \leq 7$.

Appendix III. Miscellaneous formulas. The following formulas are useful in manipulating the Coulomb functions. They may be derived from the definitions of the various functions and formulas given in Chapter 16 of Whittaker and Watson 8 . We should note that (A8) corrects an error in the relation of Example 2, p. 346 of that text. Also, (A9) was given incorrectly by Wannier and corrected by $\mathrm{Kuhn}^{5}$.

$$
\begin{aligned}
M_{n, L+1 / 2}(s)=\frac{\Gamma(2 L+2)}{\Gamma(L+1-n)} e^{i n x} W_{-n, L+1 / 2}\left(s e^{i \pi}\right) & \\
& +\frac{\Gamma(2 L+2)}{\Gamma(n+L+1)} e^{i \pi(n-L-1)} W_{n, L+1 / 2}(s) .
\end{aligned}
$$

$[(2 L+1) \neq$ negative integer $]$. 


$$
\begin{aligned}
& W_{n, L+1 / 2}\left(z^{2} / 4 n\right)=\left[\Gamma(n+L+1) n^{-L-1}(z / 2) J_{2 L+1}^{n}(z) \cos (n-L-1) \pi\right. \\
& \left.+\Gamma(n-L) n^{L}(z / 2) N_{2 L+1}^{*}(z) \sin (n-L-1) \pi\right] \text {. } \\
& (z / 2) N_{2 L+1}^{n}(z)=\left(n^{-L} e^{i \pi n}\right)(1 / \pi)\left[e^{-i \pi(L-1 / 2)} \sin \pi(n-L) \Gamma(L+1-n) W_{n, L+1 / 2}\left(z^{2} / 4 n\right)\right. \\
& \left.-\Gamma(L+1+n) \cos \pi(n-L) W_{-n, L+1 / 2}\left(\left(z^{2} / 4 n\right) e^{i \pi}\right)\right] \text {. } \\
& M_{n, L+1 / 2}(s) \underset{\text { l.1 } \sim \infty}{\sim} \frac{\Gamma(2 L+2)}{\Gamma(L+1-n)} e^{0 / 2} s^{-n}+\frac{\Gamma(2 L+2) e^{i(n-L-1) z}}{\Gamma(n+L+1)} e^{-0 / 2 s^{n}} . \\
& {[(2 L+1) \neq \text { negative integer } ;-\pi<\arg (s)<0 .]} \\
& (z / 2) N_{2 L+1}^{n}(z) \underset{|z| \rightarrow \infty}{\sim}\left(n^{-L} e^{i n \pi}\right)(1 / \pi) \\
& \text { - }\left[e^{-i \pi(L-1 / 2)} \sin \pi(n-L) \Gamma(L+1-n) \exp \left(-z^{2} / 8 n\right)\left(z^{2} / 4 n\right)^{*}\right. \\
& \left.-\Gamma(L+1+n) \cos \pi(n-L) \exp \left(z^{2} / 8 n\right) e^{-i \pi n}\left(z^{2} / 4 n\right)^{-n}\right] \text {. } \\
& {\left[-\pi<\arg \left(z^{2} / 4 n\right)<0\right] \text {. }}
\end{aligned}
$$

Appendix IV. Derivation of formulas for the repulsive field. Equations (9), (19), and (23) are valid for arbitrary complex values of the parameters $L, z$, and $n$. From (1) we see for attractive fields that if we take $r$ and $z$ positive real, then real (and for convenience, positive) values of $n$ correspond to negative energies, pure imaginary values of $n$ to positive energies. Again from (1), we see that for repulsive fields and positive energies we may conveniently choose $r$ to be real and negative and $n$ and $z$ to be negative pure imaginary numbers. We shall restrict our attention to values of $L$ that are positive integers or zero.

The regular and irregular functions, $F_{L}$ and $G_{L}$ respectively, used by Breit and others $^{1-3}$, have the asymptotic forms for fixed energy and large radius

$$
\begin{aligned}
& F_{L} \underset{\rho \rightarrow \infty}{\sim} \sin [\rho-(\pi L / 2)-\eta \ln 2 \rho+\arg \Gamma(L+1+i \eta)], \\
& G_{L} \underset{\rho \rightarrow \infty}{\sim} \cos [\rho-(\pi L / 2)-\eta \ln 2 \rho+\arg \Gamma(L+1+i \eta)] .
\end{aligned}
$$

Here in Breit's notation $\rho=|r| /|n|, \eta=|n|$, and $x=|z|=(8 \rho \eta)^{1 / 2}$. Comparing these with the asymptotic expansions of our functions for large $|r|$, got from (A11) and (A12), we find that

$$
F_{L}=\frac{|\Gamma(L+1+i \eta)| e^{-\pi \eta / 2} e^{i \pi(L+1)}}{2 i \eta^{L+1}}\left(\frac{x}{2}\right) J_{2 L+1}^{-i \eta}(-i x)
$$

and that

$$
\left(\frac{-i x}{2}\right) N_{2 L+1}^{-i \eta}(-i x)=|\Gamma(L+1+i \eta)| \eta^{-L} e^{\pi \eta / 2}(1 / \pi)\left[e^{\pi \eta-\pi L L}\left(i F_{L}\right)-e^{-\pi \eta+\pi i L} G_{L}\right] \text {. }
$$

From this latter formula we see that since both $F_{L}$ and $G_{L}$ are real, then

$$
G_{L}=\frac{(-1)^{L+1} e^{r \eta / 2} \eta^{L} \pi}{|\Gamma(L+1+i \eta)|} \text { R.P. }\left\{\left(\frac{-i x}{2}\right) N_{2 L+1}^{-i \eta}(-i x)\right\} \text {. }
$$


We can obtain the real part (R.P.) of $(-i x / 2) N_{2 L+1}^{-i n}(-i x)$ from either (19) or (23) if we make use of the formulas ${ }^{14}$

$$
\begin{aligned}
& (-i x / 2)^{m} J_{m}(-i x)=e^{-\pi i m}(x / 2)^{m} I_{m}(x), \\
& (-i x / 2)^{m} Y_{m}(-i x)=(-2 / \pi \cos \pi m)(x / 2)^{m} K_{m}(x)-i e^{-\pi i m}(x / 2)^{m} I_{m}(x) .
\end{aligned}
$$

Using (23), we evidently arrive at the asymptotic expansion of $G_{L}$ given by Breit and Hull $^{i}$, and from (19) we obtain the convergent series of Jackson and Blatt ${ }^{3}$. We have explicitly checked the terms containing $\left(1 / \eta^{2}\right)$ to the powers zero and one in our resulting expansions for $L=0$ against those given by Breit and Bouricius ${ }^{1}$ and Jackson and Blatt. The agreement is complete except that the latter authors have omitted a minus sign in the term $H_{1}(r)$ of their equation (A3.14); the sign of this term is given correctly by Breit and Bouricius.

14Ibid., pp. 372-373 